\title{
Expression of the TIMP2 gene is not regulated by promoter hypermethylation in the Caski cell line
}

\author{
GAURAV PARASHAR and NEENA CAPALASH \\ Department of Biotechnology, Panjab University, Chandigarh, India
}

Received October 26, 2011; Accepted December 28, 2011

DOI: $10.3892 / \mathrm{ol} .2012 .608$

\begin{abstract}
Promoter hypermethylation has been linked to loss of expression of tumor suppressor genes in various types of tumors. A strong reciprocal correlation between promoter hypermethylation and expression of the TIMP2 gene was observed in the Caski cell line. The TIMP2 promoter was found to be methylated within the 1919 and 1987 region $(-325$ to -257), relative to the transcription start site through methylation-specific PCR in the HeLa, SiHa and Caski cervical cancer cell lines. However, a reverse transcription PCR analysis of the TIMP2 gene confirmed a normal expression in the HeLa and $\mathrm{SiHa}$ cell lines with a high expression in the Caski cell line, indicating that expression of the TIMP2 gene is independent of methylation of $\mathrm{CpG}$ sites located within the -325 to -257 region of the TIMP 2 promoter, relative to the transcription start site.
\end{abstract}

\section{Introduction}

Cervical cancer remains one of the leading causes of cancer-related mortality in women despite medical advances and the availability of vaccination programmes (1). Promoter hypermethylation of tumor suppressor genes (TSGs) has long been contested as a probable cause of cancer and its progression (2). Several TSGs are inactivated by this mechanism (3). The tissue inhibitor of metalloproteinases-2 (TIMP2) is known to antagonize matrix metalloproteinase activity and to suppress tumor growth, angiogenesis, invasion and metastasis. The TIMP2 gene is known to be expressed in normal human tissues, whereas its expression is downregulated in glioblastomas and metastatic lung tumors (4). TIMP2 overexpression had an inhibitory effect on tumor growth and angiogenesis in a breast cancer mouse model (5). In addition, overexpression of TIMP 2 has been shown to restrict the invasiveness of various tumor cell types in vitro $(6,7)$. Suzuki et al (8) observed methylated TIMP2 in the colorectal cancer cell line

Correspondence to: Dr Neena Capalash, Department of Biotechnology, Panjab University, Sector 14, Chandigarh 160014, India

E-mail: caplash@pu.ac.in

Key words: TIMP2, promoter methylation, cervical cancer, gene expression, biomarker
RKO. However, methylation of TIMP2 was not commonly found in primary colorectal tumors. The frequency of the hypermethylated TIMP2 gene in cervical cancer was found to be $47 \%$ by Ivanova et al (9), whereas methylation-specific PCR (MSP) and sodium bisulfite analysis of genomic DNA of the HeLa cell line revealed an unmethylated promoter and expression of TIMP2. SiHa and Caski cervical cancer cell lines had a methylated promoter with downregulated expression of corresponding gene activity and methylation of the $5^{\prime}$ region of the TIMP 2 gene. Therefore, in the present study, we aimed to study the role of the promoter hypermethylation and associated TIMP 2 expression in cervical cancer cell lines.

\section{Materials and methods}

Cell culture. The cervical cancer cell lines HeLa, SiHa and Caski were procured from NCCS (Pune, India) and maintained in RPMI-1640 (Sigma, St. Louis, MO, USA) supplemented with $10 \%$ FBS (Life Technologies, Israel) and 10,000 units of penicillin and streptomycin (Sigma) at $37^{\circ} \mathrm{C}$ and $5 \% \mathrm{CO}_{2}(10)$.

Treatment of cell lines. HeLa, SiHa and Caski cell lines were treated with 5-aza-2'-deoxycytidine (Sigma) (positive control) at $20-\mu \mathrm{M}$ concentration for 4 days with change of media along with 5-aza-2'-deoxycytidine every $48 \mathrm{~h}$. Untreated cells were used as a control to analyse the promoter methylation status of the TIMP2 gene.

Methylation specific PCR (MSP). DNA was isolated using standard phenol:chloroform extraction and quantified using an ND1000 spectrophotometer (Thermo Scientific). DNA $(1 \mu \mathrm{g})$ was subjected to bisulfite modification using EZ gold methylation kit (Zymo Research). Bisulfite-modified DNA was used for MSP of the TIMP2 gene with a set of primers (9) spanning regions 1919-1987 (-325 to -257), relative to the transcription start site. MSP was performed as detailed by Ivanova et al (9). The PCR products were analysed on 3\% agarose gel. MSP was carried out in duplicate.

Reverse transcription PCR (RT-PCR). Total RNA was isolated from cultured cells using TRI reagent (Sigma) and was treated with RNAse-free DNAseI (Fermentas) to eliminate any DNA contamination. cDNA was synthesized using a First Strand Revertaid cDNA synthesis kit (Fermentas). RT-PCR was carried out for TIMP2, $\beta$-actin and GAPDH genes using the 
A

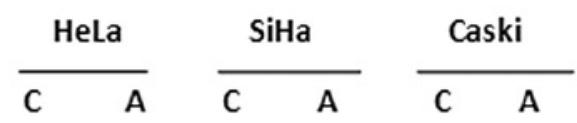

$100 \mathrm{bp}$

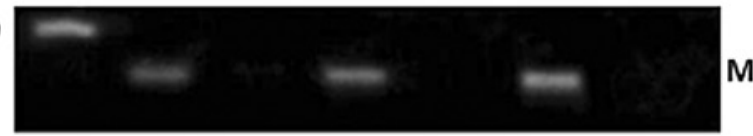

$100 \mathrm{bp}$

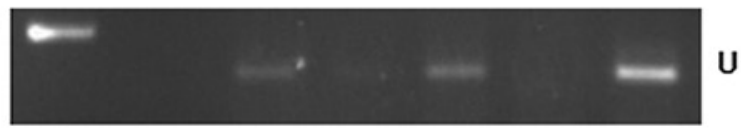

B
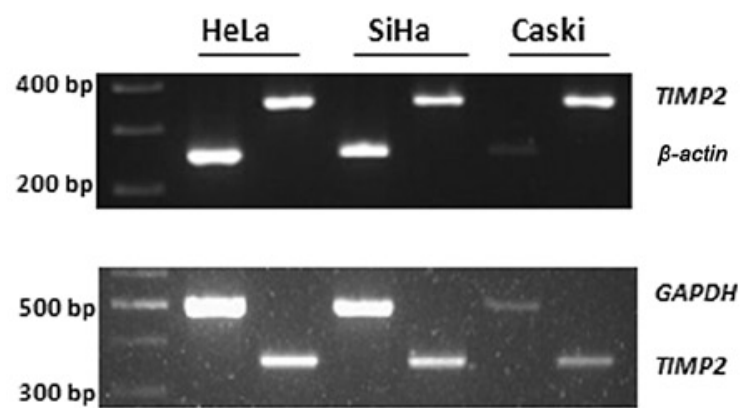

C

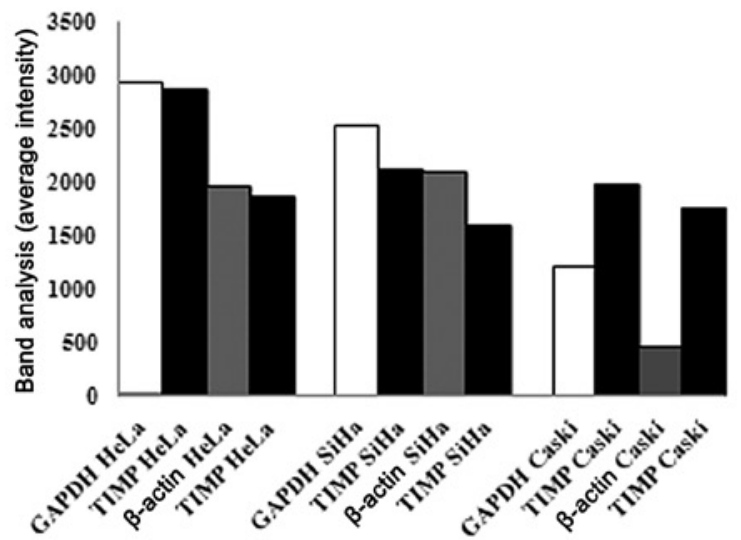

Figure 1. (A) M, methylation-specific band; U, unmethylation-specific band; C, control samples; A, 5-aza-2'-deoxycytidine treated samples. From left: lane 1, 100 bp DNA ladder; lanes 2-3, TIMP2 MSP in the HeLa cell line; lanes 4-5, TIMP2 MSP in the SiHa cell line; lanes 6-7, TIMP2 MSP in the Caski cell line. (B) RT-PCR was carried out to analyse the expression of TIMP2 in the HeLa, SiHa and Caski cell lines, respectively. From left: lane 1, 100 bp DNA ladder; lanes 2-3, comparative expression analysis of TIMP2, $\beta$-actin and GAPDH in the HeLa cell line; lanes 4-5, comparative expression analysis of TIMP2, $\beta$-actin and GAPDH in the SiHa cell line; lanes 6-7, comparative expression analysis of TIMP2, $\beta$-actin and GAPDH in the Caski cell line. (C) Comparative densitometric analysis of TIMP2 expression with $\beta$-actin and GAPDH in the HeLa, SiHa and Caski cell lines.

following primers: TIMP2 (forward: 5'-TGGCCT TTATATTTGATCCACAC-3', reverse: 5'-AAAAATCCAAAC GGAAACAAAAT-3'); $\beta$-actin (forward: 5'-CATGTACGT TGCTATCCAGGC-3', reverse: 5'-CTCCTTAATGTCACG CACGAT-3'); GAPDH (forward: 5'-CAAGGTCATCCA TGACAACTTTG-3', reverse: 5'-GTCCACCACCCTGTTGCT GTAG-3'), under the following conditions: initial denaturation at $94^{\circ} \mathrm{C}$ for $3 \mathrm{~min}$ followed by 28 cycles $\left(94^{\circ} \mathrm{C}\right.$ for $30 \mathrm{sec}, 60^{\circ} \mathrm{C}$ for $45 \mathrm{sec}, 72^{\circ} \mathrm{C}$ for $45 \mathrm{sec}$ ) and final extension at $72^{\circ} \mathrm{C}$ for 5 min. $\beta$-actin and $G A P D H$ were regarded as the internal control. Densitometric analysis of the bands was performed using 1D analysis software with average density as a parameter, calculated using intensity (INT U) $/ \mathrm{mm}^{2}$ with a sensitivity of 10 (Bio-Rad, Hercules, CA, USA). Fold change in expression was calculated for each band using the formula: Fold change $=$ average density of test gene/average density of internal control.

\section{Results}

MSP. MSP for the TIMP2 gene with primers specific to methylated DNA was carried out with untreated and 5' aza-2'-deoxycytidine treated cells, which resulted in the amplification of a 68-bp amplicon in untreated cells of the HeLa, SiHa and Caski cell lines, whereas no such band was observed in 5' aza-2'-deoxycytidine treated cells. Primers specific for unmethylated DNA responded only with samples treated with 5' aza-2'-deoxycytidine, resulting in amplification of the 68-bp amplicon (Fig. 1A).

$R T$-PCR. RT-PCR for the TIMP2 gene was carried out for the $\mathrm{HeLa}, \mathrm{SiHa}$ and Caski cell lines to assess the impact of promoter hypermethylation on the expression of the TIMP 2 gene in untreated cells. Expression of the TIMP2 gene was found to be normal in the HeLa cell line. In the case of the SiHa cell line, the TIMP2 gene exhibited downregulation, which was not found to be significant at RT-PCR level, whereas TIMP2 was found to be markedly upregulated in the Caski cell line, which was confirmed following a comparison of two internal controls (Fig. 1B). As per the analysis, the expression of the TIMP2 gene was found to be 3.7- and 1.6-fold in the Caski cell line, 0.95- and 0.98-fold in the HeLa cell line, and 0.76- and 0.83-fold (Fig. 1C) compared to $\beta$-actin and $G A P D H$, respectively.

\section{Discussion}

The TIMP2 gene, an endogenous inhibitor of matrix metalloproteinase $(M M P)$, plays a significant role in cell invasion and tumorigenesis and is downregulated or silenced in various human cancer cell lines. The percentage ratio of TIMP 2 expression compared to that of $M M P$ has always been debated. High expression of both TIMP2 and MMP 9 has been observed in invasive cancer cells of the cervix and the surrounding stromal cells, whereas the expression of both TIMP 2 and MMP9 is 


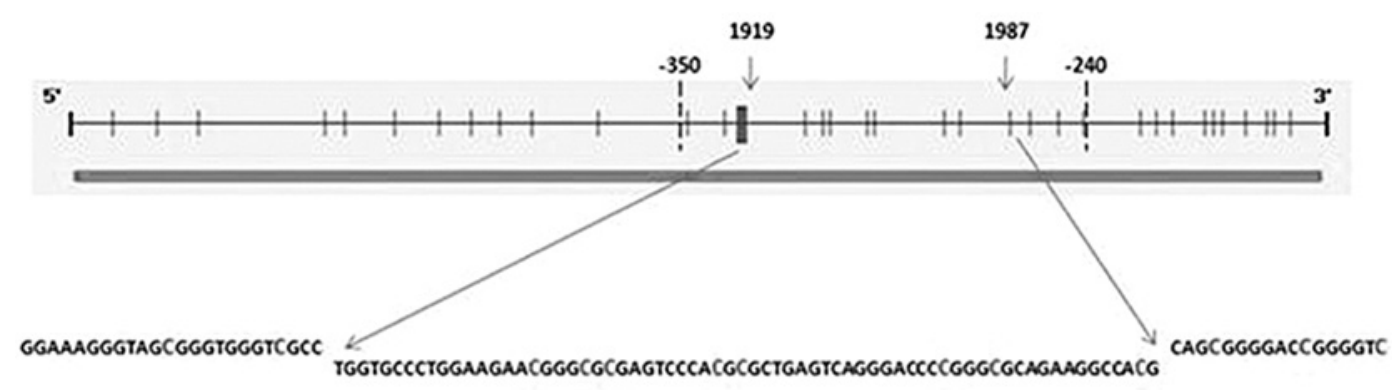

Figure 2. (I) CpG site. Eight CpG sites exist between the 1919 and 1987 regions of a 2691-bp-long sequence with the exon sequence extending from the 2244 to the 2669 region of the TIMP2 gene, exon 1 (GenBank accession number: U 44381).

hardly detectable in cervical intraepithelial neoplasias (CIN) by hybridization and in situ RT-PCR $(11,12)$. The fact that TIMP2 is an inhibitor of MMPs means that it belongs to a significant family of tumor suppressor genes whose function requires comprehensive evaluation. During our ongoing study on DNA methylation, we selected the TIMP2 gene and focused on a possible reversal of hypermethylation and subsequent reactivation.

Since there was only one published study by Ivanova et al available (9) relating TIMP2 promoter hypermethylation to cervical cancer and, specifically, cervical cancer cell lines, a TIMP2 promoter hypermethylation study spanning the 1919-1987 region, i.e., -325 to -257 relative to the transcription start site was undertaken. However, the results were very dissimilar to those already reported. The promoter of the TIMP2 gene was found to be methylated in the HeLa cell line using MSP, which was confirmed after treatment of the HeLa cell line with 5' aza-2'-deoxycytidine leading to the disappearance of the $68 \mathrm{bp}$ methylation-specific band. Primers specific for unmethylated DNA responded only to 5' aza2'-deoxycytidine treated samples, which again confirmed the promoter hypermethylation, contrary to the existing study. Similarly, methylation of the TIMP2 gene was confirmed in the SiHa and Caski cell lines. Upon meta-analysis of the TIMP2 sequence (GenBank accession number: U 44381), eight $\mathrm{CpG}$ sites were found in the 1919-1987 (-325 to -257) region and 13 $\mathrm{CpG}$ sites were found in total when the range was extended to -350 to -240 , as in the study carried out by Ivanova et al (9), relative to the transcription start site (Fig. 2). The $\mathrm{CpG}$ sites within the -350 to -240 region in the HeLa cell line were found to be unmethylated, correlating with the normal expression of the TIMP2 gene in the HeLa cell line. In the case of the $\mathrm{SiHa}$ cell line, seven $\mathrm{CpG}$ sites were hemimethylated, three were methylated and three were found to be unmethylated, whereas in the Caski cell line all CpG sites were found to be methylated with the exception of one or two sites that correlated with the downregulated gene expression of TIMP2 in the SiHa and Caski cell lines. In the present study, the transcription of the TIMP2 gene was found to be highly upregulated in the Caski cell line despite the promoter being hypermethylated. High expression in the Caski cell line was also confirmed with another housekeeping gene, GAPDH, in addition to $\beta$-actin; however, there was a consistent expression of the TIMP 2 gene in the HeLa cell line, whereas the downregulated expression was observed in the SiHa cell line despite promoter hyper- methylation, which was moderately observable at the RT-PCR level.

High expression of TIMP2 in HPV-16 positive SiHa and Caski cell lines has been reported (13) but discussion is lacking on the effect and role of promoter methylation status of the TIMP2 gene in these cervical cancer cell lines. Our study clearly showed that the CpG sites within the 1919-1987 $(-325$ to -257$)$ region, relative to the transcription start site of the TIMP2 promoter, do not regulate the expression of the TIMP2 gene in the Caski and HeLa cell lines. However, the expression in the $\mathrm{SiHa}$ cell line indicated downregulation but was not found to be significant. Five $\mathrm{CpG}$ sites still remain in the -350 to -240 region, relative to the transcription start site, of which two $\mathrm{CpG}$ sites are situated upstream of -325 and the remaining three $\mathrm{CpG}$ sites are situated downstream of -247. The methylation profile of specific $\mathrm{CpG}$ sites within a promoter, located towards the transcription start site are involved in the regulation of gene expression (14). Since the TIMP2 gene is expressed in cervical cancer cell lines, the involvement of other $\mathrm{CpG}$ sites situated downstream of -247 , including the remaining three $\mathrm{CpG}$ sites is significant. It is speculated that other $\mathrm{CpG}$ sites located towards the transcription start site play a more significant role in regulating the expression of the TIMP2 gene in cervical cancer cell lines and specifically the Caski cell line. In conclusion, we did not find a clear-cut correlation between promoter hypermethylation and expression of TIMP 2 gene in cervical cancer cell lines, indicating that the interplay of methylation and location of $\mathrm{CpG}$ sites in the promoter near the transcription site may be important for gene expression.

\section{Acknowledgements}

This study was supported by the Council of Scientific and Industrial Research (CSIR), New Delhi, India.

\section{References}

1. Guerry SL, De Rosa CJ, Markowitz LE, et al: Human papillomavirus vaccine initiation among adolescent girls in high-risk communities. Vaccine 29: 2235-2241, 2011.

2. I-Wen T, Pei-Chi H, Kuan-Der L, et al: Targeted methylation of two tumor suppressor genes is sufficient to transform mesenchymal stem cells into cancer stem/initiating cells. Cancer Res 71: 4653-4663, 2011.

3. Esteller M, Corn PG, Baylin SB, et al: A gene hypermethylation profile of human cancer. Cancer Res 61: 3225-3229, 2001. 
4. Mohanam S, Wang SW, Rayford A, et al: Expression of tissue inhibitors of metalloproteinases: negative regulators of human glioblastoma invasion in vivo. Clin Exp Metastasis 13: 57-62, 1995

5. Hajitou A, Sounni NE, Devy L, et al: Down-regulation of vascular endothelial growth factor by tissue inhibitor of metalloproteinase-2: effect on in vivo mammary tumor growth and angiogenesis. Cancer Res 61: 3450-3457, 2001.

6. DeClerck YA, Perez N, Shimada H, et al: Inhibition of invasion and metastasis in cells transfected with an inhibitor of metalloproteinases. Cancer Res 52: 701-708, 1992.

7. Valente P, Fassina G, Melchiori A, et al: TIMP-2 over-expression reduces invasion and angiogenesis and protects $\mathrm{B} 16 \mathrm{~F} 10$ melanoma cells from apoptosis. Int J Cancer 19: 246-253, 1998.

8. Suzuki H, Gabrielson E, Chen W, et al: A genomic screen for genes upregulated by demethylation and histone deacetylase inhibition in human colorectal cancer. Nat Genet 31: 141-149, 2002.

9. Ivanova T, Vinokurova S, Petrenko A, et al: Frequent hypermethylation of 5 ' flanking region of TIMP-2 gene in cervical cancer. Int J Cancer 108: 882-886, 2004.
10. Freshney RI: Culture of Animal Cells. A Manual of Basic Techniques. John Wiley \& Sons, New York, 1994.

11. Davidson B, Goldberg I, Kpopolovic J, et al: MMP-2 and TIMP-2 expression correlates with poor prognosis in cervical carcinoma: a clinicopathologic study using immunohistochemistry and mRNA in situ hybridization. Gynecol Oncol 73: 372-382, 1999.

12. Nuovo GJ, MacConnell PB, Simsir A, et al: Correlation of the in situ detection of polymerase chain reaction-amplified metalloproteinase complementary DNAs and their inhibitors with prognosis in cervical carcinoma. Cancer Res 55: 267-275,1995.

13. Silva Cardeal LB, Brohem CA, Silveira Corrêa TC, et al: Higher expression and activity of metalloproteinases in human cervical carcinoma cell lines is associated with HPV presence. Biochem Cell Biol 84: 713-719, 2006.

14. Jianduan L, Zhengyan Z, Miri B, et al: IGSF4 promoter methylation and expression silencing in human cervical cancer. Gynecol Oncol 96: 150-158, 2005. 